\title{
revista portuguesa de

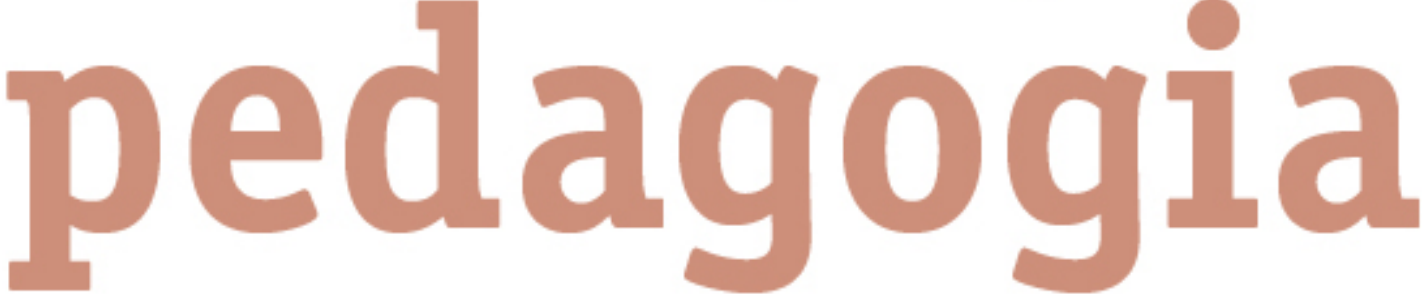

Avaliação autêntica em creche: resultados preliminares do processo de construção da ferramenta "CRECHEndo"

Autor(es): $\quad$ Carvalho, Cindy Mutschen; Portugal, Gabriela

Publicado por: Imprensa da Universidade de Coimbra

URL

persistente:

URI:http://hdl.handle.net/10316.2/41482

DOI:

DOI:https://doi.org/10.14195/1647-8614_50-2_5

Accessed : $\quad$ 26-Apr-2023 15:40:49

A navegação consulta e descarregamento dos títulos inseridos nas Bibliotecas Digitais UC Digitalis, UC Pombalina e UC Impactum, pressupõem a aceitação plena e sem reservas dos Termos e Condições de Uso destas Bibliotecas Digitais, disponíveis em https://digitalis.uc.pt/pt-pt/termos.

Conforme exposto nos referidos Termos e Condições de Uso, o descarregamento de títulos de acesso restrito requer uma licença válida de autorização devendo o utilizador aceder ao(s) documento(s) a partir de um endereço de IP da instituição detentora da supramencionada licença.

Ao utilizador é apenas permitido o descarregamento para uso pessoal, pelo que o emprego do(s) título(s) descarregado(s) para outro fim, designadamente comercial, carece de autorização do respetivo autor ou editor da obra.

Na medida em que todas as obras da UC Digitalis se encontram protegidas pelo Código do Direito de Autor e Direitos Conexos e demais legislação aplicável, toda a cópia, parcial ou total, deste documento, nos casos em que é legalmente admitida, deverá conter ou fazer-se acompanhar por este aviso. 


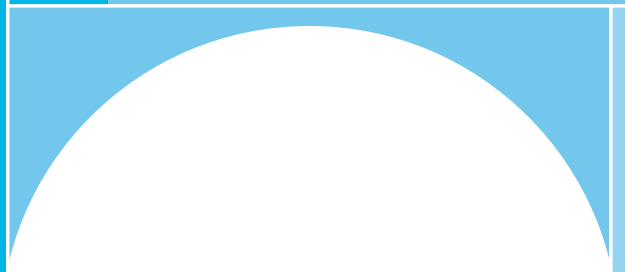

\section{revista portuguesa de pedagogia}




\title{
Avaliação Autêntica em Creche: Resultados Preliminares do Processo de Construção da Ferramenta "CRECHEndo"
}

\author{
Cindy Mutschen Carvalho ${ }^{2}$ e Gabriela Portugal ${ }^{3}$
}

\begin{abstract}
Resumo
Este estudo assenta sobre os fundamentos teóricos da educação experiencial e parte da análise do contexto sociopolítico da creche em Portugal. O objeto de estudo é uma ferramenta de avaliação para o contexto de creche (CRECHEndo), direcionada a educadores de infância, tendo em vista a melhoria da qualidade das práticas educativas. Esta ferramenta, de natureza processual, serve de ponto de partida para a observação, reflexão e planificação curricular. Apresentam-se resultados preliminares de vários estudos de caso desenvolvidos em 2013/2014, partindo do acompanhamento da aplicação do CRECHEndo por educadores de duas creches portuguesas. Os resultados evidenciam tendências gerais na avaliação do bem-estar e implicação das crianças e temas dominantes na análise dos fatores contextuais que afetam estas variáveis. Reconhecendo a ainda pouca profundidade dos resultados apresentados, estes primeiros dados apontam para o potencial do CRECHEndo enquanto ferramenta de apoio à reflexão e planificação dos educadores de infância a trabalhar em creche.
\end{abstract}

Palavras-chave: avaliação autêntica; creche; CRECHEndo; práticas educativas

\footnotetext{
1 Apoio financeiro da FCT - Fundação para a Ciência e Tecnologia no âmbito do QREN - POPH - Tipologia 4.1 - Formação Avançada, comparticipado pelo Fundo Social Europeu e por fundos nacionais do MEC (SFRH/BD/87555/2012).

2 Universidade de Aveiro/CIDTFF. Email: cindymutschen@ua.pt

3 Universidade de Aveiro/CIDTFF. Email: gabriela.portugal@ua.pt

Artigo recebido a 23-10-2015 e aprovado a 16-12-2016.
} 


\title{
Authentic Assessment in Infant Daycare: Preliminary Results of the "CRECHEndo" Tool Construction Process
}

\begin{abstract}
This study is based on the theoretical foundations of experiential education and on the analysis of the socio-political context of infant daycare in Portugal The object of study is an evaluation tool for daycare (CRECHEndo), aimed at kindergarten teachers, which aims to improve the quality of educational practices. This procedural tool is the starting point for observation, reflection and curriculum planning. We present preliminary results from several case studies developed in 2013/2014, based on the monitoring of the CRECHEndo implementation by educators of two Portuguese infant daycare centers. The results show general trends in assessing the wellbeing and involvement of children and dominant themes in the analysis of the contextual factors that affect these variables. Recognizing the still shallow depth of the results, these first data suggest the potential of CRECHEndo as a support tool for reflection and planning of early childhood educators working in daycare.
\end{abstract}

Keywords: authentic assessment; infant daycare; CRECHEndo; educational practices

\section{Evaluación Auténtica en la Guardería: Resultados Preliminares del Proceso de Construcción de la Herramienta "CRECHEndo"}

\section{Resumen}

Este estudio se basa en los fundamentos teóricos de la educación experiencial y parte del análisis del contexto socio-político de la guardería en Portugal. El objeto de estudio es una herramienta de evaluación para el contexto de la guardería (CRECHEndo), dirigido a los maestros de educación infantil, con miras a mejorar la calidad de las prácticas educativas. Esta herramienta es el punto de partida para la observación, la reflexión y la planificación del currículo. Se presentan los resultados preliminares de varios estudios de caso desarrollados en 2013/2014, basado en el seguimiento de la aplicación de CRECHEndo por maestros de dos guarderías portuguesas. Los resultados muestran tendencias generales de la evaluación del bienestar y la implicación de los niños y los temas dominantes en el análisis de los factores contextuales que afectan estas variables. Reconociendo todavía la pequeña profundidad de los resultados, estos primeros datos apuntan a la posibilidad de lo CRECHEndo como herramienta de apoyo para la reflexión y la planificación de los educadores de la primera infancia que trabajan en la guardería. 


\section{Introdução}

Os resultados preliminares apresentados neste artigo enquadram-se no âmbito de um projeto de doutoramento ainda em curso. O objetivo geral desse trabalho prende-se com a testagem, aperfeiçoamento e divulgação de uma ferramenta de avaliação das práticas educativas em creche, tendo como pano de fundo a questão geral "como promover a melhoria das práticas educativas em creche partindo da avaliação e reflexão?"

Neste artigo, abordaremos apenas um dos objetivos específicos dessa investigação mais ampla - testar a ferramenta de avaliação (denominada CRECHEndo) na prática (com a colaboração de educadores de infância), avaliando as suas potencialidades e fragilidades. Assim, neste texto, pretendemos analisar a avaliação da implicação e do bem-estar das crianças realizada por um conjunto de educadores de infância e identificar os temas gerais emergentes na sua análise do grupo e do contexto (em contraponto com a visão da investigadora).

Com vista a enquadrar teoricamente o nosso estudo, iniciamos o artigo com um breve enquadramento sobre a creche em Portugal e sobre a qualidade e avaliação em educação de infância, com destaque para a perspetiva avaliativa da educação experiencial. Segue-se um descritivo da metodologia utilizada neste estudo, a apresentação dos resultados e sua discussão. Nas considerações finais, procuramos delinear as principais potencialidades e fragilidades do estudo desenvolvido.

\section{A creche em Portugal}

A creche é um equipamento de natureza socioeducativa, vocacionado para o apoio à família e à criança, que acolhe crianças até aos três anos de idade, durante o período correspondente ao impedimento dos pais ou outros responsáveis. As atividades e serviços prestados envolvem cuidados adequados à satisfação das necessidades das crianças, cuidados na nutrição e higiene pessoal, atendimento individualizado, atividades pedagógicas, lúdicas e de motricidade e disponibilização de informação à família (Portaria n. ${ }^{\circ}$ 262/2011 de 31 de agosto).

Em Portugal, a tutela dos cuidados e educação para crianças até aos três anos pertence ao Ministério do Trabalho, Solidariedade e Segurança Social, o que significa que os serviços destinados a estas crianças têm uma função essencialmente assistencial e não são uma opção clara pelo "direito à educação" (UNICEF, 1989). Ao contrário do que tem acontecido com a Educação Pré-Escolar (3-6 anos), não 
tem havido políticas educativas claras e sistemáticas na área da creche (Taguma, Lijtens, \& Makowiecki, 2012; Vasconcelos, 2011).

O Perfil Específico de Desempenho Profissional do Educador de Infância menciona a possibilidade de a sua formação o habilitar para outras funções educativas, nomeadamente junto de crianças com menos de três anos (Decreto-Lei $n^{\circ}$ 241/2001 de 30 de agosto). No entanto, a formação continua a privilegiar o trabalho com crianças a partir dos três anos, tal como é apontado pela OCDE, que critica a falta de formação específica para os que trabalham com as crianças mais novas (Araújo, 2012a; Craveiro, 2011; OCDE, 2014).

Vários estudos portugueses têm demonstrado que os serviços de atendimento à primeira infância (até aos três anos) apresentam fortes lacunas e níveis baixos de qualidade (Aguiar, Bairrão, \& Barros, 2002; Barros, 2007; Barros \& Aguiar, 2010), sendo que a falta de formação específica dos adultos responsáveis é um dos fatores apontados como explicação para estes resultados.

Se considerarmos que as experiências nos primeiros anos de vida são cruciais para a aprendizagem e desenvolvimento futuros (Shonkoff \& Phillips, 2000; Vasconcelos, 2011), facilmente se compreende a importância de os profissionais encararem a creche como um espaço educativo e não apenas de cuidado (Coelho, 2004). Para que tal seja possível, torna-se fundamental que existam orientações pedagógicas que permitam aos profissionais a monitorização e o aperfeiçoamento das suas práticas com base na observação e reflexão. Como referem Góis e Portugal (2009, p. 29), a "reflexão crítica, o questionamento de práticas e o trabalho de equipa não [são] suficientemente implementados" em contextos de educação de infância, gerando uma acomodação que prejudica a qualidade das intervenções e contribui para que o processo de intencionalidade educativa se limite à alusão a alguns aspetos do desenvolvimento (Coelho, 2004).

Vasconcelos (2009) sintetiza alguns dos principais problemas existentes no campo da educação de infância: "a inexistência de supervisão pedagógica e de regulação da qualidade dos estabelecimentos; a não avaliação dos educadores e o seu fechamento em estruturas que os isolam" (p. 20). O esforço de implementação e desenvolvimento de práticas de qualidade deve ser acompanhado de iniciativas no campo da avaliação e supervisão dos profissionais. Neste âmbito, os projetos de formação contínua em contexto de trabalho (Araújo, 2013) e centrados nas práticas (Estrela \& Estrela, 2001; Nóvoa, 1991) constituem instrumentos importantes para o aperfeiçoamento e melhoria da qualidade dos serviços.

Todos estes aspetos apontam para uma grande necessidade de desenvolver iniciativas que promovam a qualidade na creche. 


\section{Qualidade e avaliação em educação de infância}

Distinguem-se, habitualmente, duas vertentes da qualidade, interrelacionadas: a qualidade estrutural (incluindo variáveis passíveis de regulamentação como o rácio adulto-criança, o tamanho do grupo e as características do educador em termos de formação, experiência e estabilidade no grupo) e a qualidade processual (incluindo a existência de atividades adequadas ao desenvolvimento, o carinho, riqueza e sensibilidade dos cuidados prestados, etc.) (Barros, 2007; Cryer, 1999; Vandell \& Wolfe, 2000). Existem, porém, diferentes formas de considerar os processos, podendo remeter-nos, por um lado, para as interações entre adultos e crianças (Aguiar, Bairrão, \& Barros, 2002; Barros, 2007; Barros \& Aguiar, 2010) ou, noutra perspetiva, para as experiências internas da criança, lidas em termos dos seus níveis de implicação e bem-estar (Laevers, 2014; Portugal \& Laevers, 2010; Santos \& Portugal, 2002).

A relação entre avaliação e qualidade é complexa, mas, simultaneamente, intuitiva - se a qualidade remete para algo ideal, que pretendemos atingir, podemos encarar a avaliação como o processo de controlo e monitorização que nos acompanha ao longo do percurso até esse ideal (ou resultado).

A realidade da avaliação realizada em contextos de educação de infância deverá assumir uma dimensão marcadamente formativa (Castilho \& Rodrigues, 2013; Lopes da Silva, 2013), num "processo dinâmico em que o educador procura os meios de facilitar a aprendizagem da criança, em vez de verificar as suas capacidades num determinado momento" (Lopes da Silva, 2013, p. 161).

Embora na literatura se defenda um processo de reflexão, acompanhamento e avaliação que permita uma constante melhoria das práticas (Hoffman, 2013; Portugal, 2013) e uma avaliação processual e autêntica (Portugal, 2013), muitos educadores de infância terão lacunas nesta área, confundindo observação informal com avaliação (Lopes da Silva, 2013). Por um lado, o facto de terem muito conhecimento sobre as crianças, e de passarem muito tempo com elas, leva muitas vezes os educadores a optar por uma avaliação assistemática e superficial, baseada na observação informal e não intencional realizada no quotidiano (Lopes da Silva, 2013). Por outro lado, os educadores de infância portugueses usarão, muitas vezes, procedimentos estandardizados de avaliação, recorrendo a checklists de capacidades isoladas, numa perspetiva pouco autêntica (Oliveira \& Gaspar, 2004; Portugal, 2012b, 2013).

Contrariando estas formas de avaliação tradicionais mais comuns, Bagnato e Neisworth (2007) consideram que as avaliações nos contextos de infância têm de ser desenvolvidas de forma autêntica e genuína, ou seja, nos ambientes naturais das crianças, por parte de adultos significativos, de forma contínua e em estreita colaboração com a família (Bagnato \& Neisworth, 2007; Gullo, 2014; Moreno \& Klute, 2011). 
Nesta ordem de ideias, importa optar por perspetivas teóricas valorizadoras das experiências vividas pelas crianças, articuladas com formas de avaliação mais flexíveis, contextualizadas e autênticas, na linha da educação experiencial, cuja perspetiva avaliativa se apresenta de seguida e que serviu de enquadramento a este projeto.

\section{A perspetiva avaliativa da educação experiencial}

Uma vez que "as questões de avaliação estão imbricadas com as questões que teoricamente as antecedem - as questões de filosofia educacional - e plasmam-se no modelo curricular adotado" (Oliveira-Formosinho, 2002, p. 146), é lógico considerar que propor um sistema de avaliação é, necessariamente, comprometer-se com uma abordagem pedagógica. Uma vez que este trabalho assenta, essencialmente, nos princípios propostos pela educação experiencial (EXE), importa apresentar de forma breve a perspetiva de qualidade e avaliação assumida por esta abordagem, especialmente no que diz respeito à educação de infância.

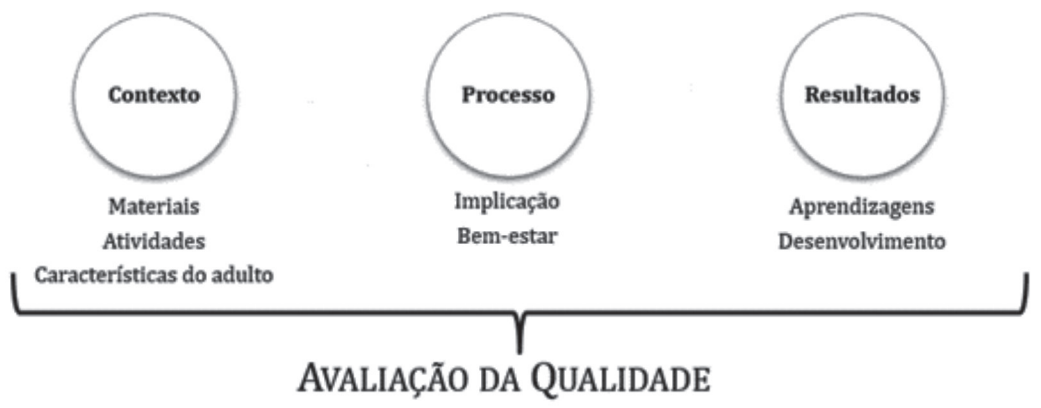

Figura 1. Modelo de Avaliação da Qualidade segundo a Educação Experimental. Adaptado de Laevers (1997, 2000, 2005c, 2014).

A EXE concebe a qualidade como um conceito multidimensional que engloba três tipos de variáveis - de contexto, de processo e de resultado (cf. Figura 1). Por permitirem um acompanhamento contínuo e uma definição de objetivos e iniciativas mais fundamentada e atempada e expressarem a forma como as crianças experienciam o contexto educativo no "aqui e agora", as variáveis processuais merecem destaque (Laevers, 1997).

A educação experiencial valoriza a experiência interna das crianças, lida através das variáveis processuais - bem-estar e implicação. Genericamente, o bem-estar 
caracteriza-se pela presença de prazer, alegria, tranquilidade, vitalidade e energia, e pela ausência de desconforto, tensão, frustração, desconfiança, agressividade, isolamento/fechamento. A implicação caracteriza-se pela motivação, persistência, satisfação, ímpeto exploratório e concentração, e pela ausência de passividade, aborrecimento, desmotivação e desinteresse. Conhecer o bem-estar e a implicação fornece feedback sobre a qualidade da atividade ou situação, atendendo ao modo como estas são vivenciadas por cada criança. É, portanto, uma abordagem intrinsecamente inclusiva, uma vez que não se foca nas competências, mas na interação atual da criança com o que a rodeia (Laevers, 2014; Portugal \& Laevers, 2010; Santos \& Portugal, 2002).

Laevers considera que a implicação, muito relacionada com o bem-estar emocional, é um parâmetro essencial da qualidade, que tem possibilidade de se manifestar quando a exigência e o desafio da atividade e as capacidades da pessoa estão equilibradas (Laevers, Moons, \& Declercq, 2012; Santos \& Portugal, 2002).

Esta abordagem tem sido adotada em vários países, através da criação e adaptação de diversas ferramentas de avaliação (Bertram \& Pascal, 2009; Laevers, 2005b; Laevers et al., 2012; Pascal \& Bertram, 2003; Portugal \& Laevers, 2010) e inúmeros estudos mostram que a observação do bem-estar e da implicação, acompanhada de uma reflexão sobre os fatores relevantes do contexto, permitem alcançar mudanças fundamentais, oferecendo um enquadramento para as práticas e para a definição de iniciativas conducentes à melhoria da qualidade (Araújo, 2012b; Kalliala \& Tahkokallio, 2003; Laevers \& Declercq, 2011; Laevers, 2000, 2005a; Ulich \& Mayr, 2003; Van Sanden \& Joly, 2003).

Na Finlândia, Kalliala e Tahkokallio (2003) acompanharam um grupo de educadores que utilizaram a avaliação da implicação das crianças, entre outros registos narrativos e entrevistas. As autoras verificaram mudanças positivas no processo reflexivo dos educadores e na implicação das crianças ao longo do processo.

Um outro estudo relevante foi desenvolvido entre 2009 e 2010 numa cidade inglesa, Milton Keynes. Após um período de treino para os educadores e para a equipa consultora, o SICS [Ziko] - A process-oriented Self-evaluation Instrument for Care Settings (Laevers, 2005b) foi utilizado como ferramenta de monitorização. A equipa consultora realizou três observações ao longo do projeto, recolhendo dados sobre mais de 1400 crianças, 53 grupos e 49 instituições (Laevers \& Declercq, 2011). Os resultados, tanto quantitativos como qualitativos, mostraram melhorias significativas nos níveis de bem-estar e implicação das crianças ao longo do projeto, tendo-se verificado que os fatores contextuais têm um grande peso nas variáveis processuais, especialmente na implicação. Estes fatores contextuais envolvem a oferta educativa (materiais, infraestruturas, atividades, 
etc.), o clima de grupo (relações entre crianças, com os adultos, com as famílias, ambiente da sala, etc.), o espaço para a iniciativa (participação das crianças nas rotinas e na organização, possibilidade de escolha livre de brincadeiras, etc.), a organização (relações entre profissionais, distribuição de tarefas, funcionamento das rotinas, etc.) e o estilo do adulto (sensibilidade, estimulação, espaço para a autonomia, etc.). A principal conclusão retirada pelos autores é que a abordagem orientada pelo processo e os seus instrumentos têm resultados positivos, oferecendo um enquadramento para as práticas e para a definição de iniciativas para a melhoria da qualidade. Os conceitos e instrumentos foram considerados de fácil acesso pelos educadores. Em suma, Laevers e Declercq (2011) consideram que o esforço para alcançar maiores níveis de bem-estar e de implicação está ao alcance de qualquer instituição de educação de infância. A observação do bem-estar e implicação, acompanhada de uma reflexão sobre os fatores contextuais, permite alcançar mudanças fundamentais em direção a um serviço de maior qualidade.

\section{Metodologia}

Como já foi mencionado, o conteúdo deste artigo relaciona-se com um dos objetivos específicos de um estudo mais amplo, relacionado com a testagem de uma nova ferramenta avaliativa para o contexto de creche, especificamente, a sua utilização para a avaliação do bem-estar e implicação das crianças e para a análise do contexto educativo. Pretende-se averiguar o conteúdo emergente da avaliação realizada pelos educadores de infância a partir desta nova ferramenta.

O processo de construção desta ferramenta (CRECHEndo) iniciou-se em 2012, num projeto de mestrado (Carvalho, 2012), partindo dos fundamentos teóricos do modelo de qualidade da Educação Experiencial, do currículo High-Scope e atendendo a finalidades educativas definidas para a Creche (Portugal, 2012a). Estas, entretanto, foram integradas no documento Orientações Pedagógicas para a Creche solicitado pelos Ministérios da Educação e do Trabalho, Solidariedade e Segurança Social (Portugal, Bento, \& Carvalho, 2016). Segue a mesma linha de pensamento de outras ferramentas enraizadas na perspetiva experiencial, como o SAC - Sistema de Acompanhamento das Crianças (Portugal \& Laevers, 2010), o POMS - Process-Oriented Monitoring System for early years (Laevers, Moons, \& Declercq, 2012) e o SICS (Laevers, 2005b).

À semelhança desses instrumentos, o CRECHEndo é composto por diferentes fichas que devem ser preenchidas pelos educadores de infância, num processo 
cíclico contínuo de observação, reflexão e ação com foco no bem-estar, implicação, aprendizagem e desenvolvimento das crianças. Trata-se de uma ferramenta essencialmente descritiva e narrativa, exigindo observação atenta e reflexão continuada por parte dos educadores que devem prestar atenção ao funcionamento do grupo de crianças através de um arco de avaliação processual e contextual, análise e definição de objetivos consequentes a integrar no Projeto Pedagógico do Grupo. A avaliação do grupo assenta na indicação dos níveis de bem-estar e implicação das crianças e sua análise com base em fatores contextuais, destacados pela educação experiencial: oferta educativa, clima de grupo, espaço para a iniciativa, organização do contexto e estilo do adulto. Os objetivos e iniciativas concretas a desenvolver são definidos a partir desta análise, considerando os fatores contextuais que poderão ser mais bem investidos com vista à melhoria da qualidade das experiências vividas pelas crianças na creche. Finalmente, o CRECHEndo prevê, ainda, análises individualizadas das crianças exigindo, mais uma vez, uma sequência de avaliação, análise e definição de objetivos, mas, desta vez, para cada uma das crianças. Estas análises individuais baseiam-se, essencialmente, na consideração das finalidades e objetivos educativos definidos para a creche (Portugal et al., 2016; Portugal, 2012a).

No âmbito do projeto de doutoramento referido, foram desenvolvidos oito estudos de caso em duas creches do distrito de Aveiro durante o ano letivo 2013/2014. Foi dado destaque, essencialmente, à análise descritiva e interpretativa das práticas de planificação e avaliação de cada um dos educadores de infância ao longo de um processo de acompanhamento que seguiu os moldes de uma formação em contexto de natureza participativa (Araújo, 2012a; Oliveira-Formosinho, 2001; Vieira \& Sarmento, 2009).

\section{Técnicas de recolha e análise de dados}

A principal técnica de recolha de dados utilizada para este artigo foi a recolha documental das fichas do CRECHEndo preenchidas pelas educadoras, em particular as respeitantes à avaliação do grupo e análise do grupo e do contexto.

A ficha de avaliação geral do grupo consiste numa grelha onde o educador é convidado a assinalar o nível de bem-estar e de implicação de cada criança do grupo, numa escala de 1 a 3 - Baixo, Médio ou Alto (cf. Figura 2). Ainda, existem duas colunas onde devem ser assinaladas as atividades ou situações em que a criança evidencia maiores e menores níveis de bem-estar e uma coluna para comentários adicionais. Os critérios para a avaliação destas duas variáveis estão descritos e exemplificados no manual de instruções do CRECHEndo. 


\begin{tabular}{|c|c|c|c|c|c|c|c|c|c|}
\hline \multirow{2}{*}{ Nomes } & \multicolumn{3}{|c|}{ Bem-estar } & \multicolumn{3}{|c|}{ Implicação } & \multirow{2}{*}{$\begin{array}{l}\text { Implica-se } \\
\text { muito em... }\end{array}$} & \multirow{2}{*}{$\begin{array}{l}\text { Não se implica } \\
\text { muito em... }\end{array}$} & \multirow{2}{*}{ Comentários } \\
\hline & Baixo & Médio & Alto & Baixo & Médio & Alto & & & \\
\hline \multicolumn{10}{|l|}{ Ana } \\
\hline \multicolumn{10}{|l|}{ Henrique } \\
\hline$\cdots$ & & & & & & & & & \\
\hline
\end{tabular}

Figura 2. Ficha de avaliação geral do grupo.

A ficha de análise e reflexão em torno do grupo e do contexto (cf. Figura 3) contempla três campos: a análise do grupo, a análise do contexto e a reflexão geral e ideias para o desenvolvimento do Projeto Pedagógico do Grupo. Na análise do contexto, o educador deve considerar o que mais lhe agrada e o que mais o preocupa relativamente aos cinco fatores contextuais já mencionados, a propósito do estudo desenvolvido por Laevers e Declercq (2011): a oferta educativa, o clima de grupo, o espaço para a iniciativa, a organização e o estilo do adulto.

Estas duas fichas estão fortemente articuladas, uma vez que, na segunda, se pretende encontrar justificações que possam explicar os altos ou baixos níveis de bem-estar e implicação assinalados na primeira. Esta análise permitirá, posteriormente, delinear objetivos e iniciativas que permitam elevar os níveis de bem-estar e implicação do grupo, elevando a qualidade das práticas educativas em creche.

\begin{tabular}{|c|c|}
\hline \multicolumn{2}{|c|}{ 1. Análise do grupo } \\
\hline \multicolumn{2}{|c|}{ O que me agrada } \\
\hline \multicolumn{2}{|c|}{ O que me preocupa } \\
\hline \multicolumn{2}{|c|}{ 2. Análise do contexto } \\
\hline \multicolumn{2}{|c|}{ Oferta } \\
\hline O que me agrada & O que me preocupa \\
\hline \multicolumn{2}{|c|}{ Clima de grupo } \\
\hline O que me agrada & o que me preocupa \\
\hline \multicolumn{2}{|c|}{ Espaço para a iniciativa } \\
\hline O que me agrada & O que me preocupa \\
\hline \multicolumn{2}{|c|}{ Organização } \\
\hline O que me agrada & $\mathrm{O}$ que me preocupa \\
\hline \multicolumn{2}{|c|}{ Estilo do adulto } \\
\hline o que me agrada & o que me preocupa \\
\hline
\end{tabular}

Figura 3. Estrutura da ficha de análise e reflexão em torno do grupo e do contexto. 
Adicionalmente, com vista a colocar em confronto os registos e relatos das educadoras com a perspetiva externa da investigadora, foram utilizados alguns registos de observação, partindo da observação naturalista (narrativa) e do preenchimento (por parte da investigadora) das duas fichas do CRECHEndo atrás mencionadas.

Todos os dados descritivos foram analisados com recurso ao software online webQDA ${ }^{\odot}$. Os dados numéricos recolhidos na avaliação do bem-estar e implicação foram organizados e tratados com recurso ao software Microsoft Excel $2013^{\circ}$ (versão 15.0.4719.1000), parte do Microsoft Office 365 ProPlus.

\section{Participantes}

Três das educadoras trabalham numa Instituição Particular de Solidariedade Social (IPSS) do distrito de Aveiro, numa pequena localidade rural, sendo responsáveis por um grupo de dois anos (crianças nascidas entre janeiro e dezembro de 2011), um grupo heterogéneo (crianças nascidas entre maio de 2012 e janeiro de 2013) e um grupo de um ano em acumulação com o cargo de direção técnica (crianças nascidas entre janeiro e julho de 2012). As restantes cinco educadoras trabalham numa IPSS do distrito de Aveiro em contexto urbano, sendo responsáveis por dois grupos de um ano (crianças nascidas entre janeiro e dezembro de 2012), dois grupos de dois anos (crianças nascidas entre janeiro e dezembro de 2011) e dois berçários (crianças nascidas entre janeiro e dezembro de 2013). A idade das educadoras varia entre os 24 e os 40 anos. Os grupos de dois anos têm entre 13 e 18 crianças, os grupos de um ano têm 12 ou 13 crianças, o grupo heterogéneo tem oito crianças e os berçários têm 13 e 11 crianças.

\section{Considerações éticas}

Em todos os momentos, foram valorizados os princípios da privacidade, anonimato e consentimento informado. As participantes foram informadas de que poderiam desistir da colaboração no estudo a qualquer momento (o que veio a acontecer com uma das participantes) e foram auscultadas sobre as melhores estratégias para garantir a sua privacidade e anonimato. Cada participante pôde gerir o processo em função da sua disponibilidade e foi-lhe comunicado que cada etapa se desenrolaria de modo a integrar-se, o melhor possível, nas suas rotinas de trabalho. Ainda, uma preocupação fundamental ao longo da investigação foi a necessidade de que o processo de investigação fosse útil para a prática dos participantes, na linha do que refere Vasconcelos (1997, p. 65), "a investigadora deve perguntar a cada passo de que maneira poderá a sua investigação ser útil para as pessoas estudadas". 


\section{Resultados e discussão}

Os resultados apresentados referem-se apenas às avaliações do bem-estar e implicação e à análise dos fatores contextuais (das fichas Avaliação geral do grupo e Análise e reflexão em torno do grupo e do contexto).

\section{Avaliação do grupo}

Os níveis de bem-estar e implicação atribuídos, tanto pelas educadoras como pela investigadora apontam para valores médios-altos, o que sugere uma relação positiva entre as crianças e o contexto. No entanto, mais do que atender aos valores absolutos, importa salientar que a avaliação do bem-estar e da implicação reflete algumas das tendências encontradas na literatura (Araújo, 2012a; Estrela, 2008; Laevers \& Declercq, 2011; Laevers et al., 2012; Machado, 2014; Nabuco \& Prates, 2003; Ulich \& Mayr, 2003), a saber:

- As educadoras atribuem níveis mais elevados de implicação e bem-estar do que um observador externo.

- Os níveis de bem-estar são ligeiramente mais elevados do que os de implicação.

Os valores mais relevantes encontram-se sintetizados no Quadro 1.

Quadro 1

Médias dos Níveis de Bem-Estar e Implicação (Níveis entre 1 - Baixo e 3 - Alto).

\begin{tabular}{lcc}
\hline & Bem-estar & Implicação \\
\hline Média - educadoras & 2.61 & 2.44 \\
Média - investigadora & 2.28 & 2.02 \\
\hline
\end{tabular}

\section{Análise do grupo e do contexto}

Quanto aos fatores contextuais que, segundo as educadoras do estudo, afetarão os níveis de implicação e de bem-estar experienciados pelas crianças, a análise de conteúdo fez emergir alguns temas facilmente identificáveis. De um modo geral, o que mais agrada às educadoras ao nível da oferta educativa é a existência de atividades e materiais diversos e estimulantes, que respondem aos interesses e necessidades das crianças. Por outro lado, e de alguma forma paradoxalmente, o que mais as preocupa é a falta de materiais e de determinadas estruturas fixas ou mobiliário. Uma explicação para estas respostas contraditórias pode estar na diferença de nível colocada 
na análise: quando se referem às preocupações, as educadoras identificam de forma mais específica e concreta os objetos que estão em falta (por exemplo, livros ou andarilhos); quando mencionam os aspetos positivos, a sua análise é mais genérica, no sentido de uma apreciação global da qualidade dos materiais e infraestruturas.

No clima de grupo, o mais valorizado é o espírito de partilha e o estabelecimento de relações positivas entre crianças. O que mais preocupa todas as educadoras são os conflitos e agressões entre pares. Este segundo aspeto coincide com as nossas observações: os conflitos e agressões são muito frequentes e geram situações de stresse intenso, tanto para os adultos como para as crianças. Com frequência, as crianças "conflituosas" são repreendidas pelos seus atos e a intervenção do adulto surge no sentido de pôr fim ao conflito, afastando as crianças ou retirando o objeto causador do conflito. Nestes momentos, há por vezes tentativas de "reconciliação", pedindo-se às crianças que peçam desculpas ou se abracem/beijem, mas não é usual procurar perceber a sequência temporal dos acontecimentos, nem tentar explicar porque a agressão constitui um problema. Retirando os momentos de conflito, as relações entre as crianças são, geralmente, positivas, o que parece ser consequência natural da sua convivência diária, ao longo da qual se vão estabelecendo parceiros preferenciais de brincadeira. As atividades alternam, na grande maioria dos casos, entre momentos de grande grupo - dirigidos pelo adulto (e.g., uma história) - e momentos de brincadeira livre. No segundo caso, o adulto raramente intervém, a menos que seja solicitado pelas crianças (para ver, pedir ajuda ou para solucionar conflitos). Embora o discurso das educadoras invoque com frequência as necessidades e interesses de cada criança e a sua identidade individual, os momentos de atenção individualizada são muito raros na rotina diária, mesmo nos momentos de cuidado. Nas mudas de fralda, higiene e refeição, os adultos procuram realizar as tarefas o mais rapidamente possível, por vezes, olhando em volta para controlar o grupo, de forma a reduzir a duração destes momentos ao mínimo.

No espaço para a iniciativa, as educadoras valorizam a possibilidade de as crianças poderem escolher livremente os materiais e atividades e também a sua participação em rotinas como a arrumação da sala, vestir/despir, etc. Quanto ao que mais preocupa, as respostas apresentam uma diversidade maior. Alguns dos aspetos mencionados são a falta de materiais que abram mais o leque de escolha das crianças, alguma dependência face ao adulto e também alguma imposição do adulto que limita a iniciativa. As nossas observações apontam para uma generalizada falta de espaço para a iniciativa. Nos momentos de brincadeira livre, as crianças podem escolher com o que querem brincar, dentro do leque de ofertas do espaço, que é, geralmente, limitado e igual, dia após dia. Mesmo dentro destas escolhas, a iniciativa da criança é, muitas vezes reprimida, colocando-se obstáculos à sua ação (e.g., no 
espaço exterior, ao tentarem mudar os pneus de lugar, as crianças são avisadas de que não é permitido). Estas situações desvalorizam a criança enquanto aprendiz ativo e competente, colocando-a sob a dependência de um adulto que controla e impõe - as respostas das educadoras afloram, à superfície, estes problemas, sem, contudo, os apontarem de forma clara.

Em relação à dimensão organização do contexto ressaltam as boas relações dentro da equipa de sala e a divisão eficaz das tarefas. No entanto, o que mais preocupa as educadoras são os desentendimentos com algumas colegas ou o sentimento de desvalorização do seu trabalho (em particular fora da equipa de sala). Um aspeto referido por diversas educadoras é a existência de rotinas confusas e desorganizadas que acabam por levar a demasiados tempos de espera (ou tempos mortos). Este último aspeto é por demais evidente em todos os contextos. De uma forma geral, os períodos de higiene, sono e refeição traduzem-se em momentos caóticos e ruidosos, sendo aqui precisamente que surgem a maioria dos conflitos atrás mencionados. Por uma questão de organização dos grupos e da instituição, as crianças de um grupo vão todas almoçar e lanchar ao mesmo tempo, tendo depois cada uma de esperar pela sua vez para lavar as mãos e mudar a fralda (quando é o caso) antes de se deitar, o que gera longos tempos de espera. A estratégia adotada na maioria dos casos consiste em juntar as crianças num espaço (e.g., corredor) e, enquanto o educador as tenta distrair do sono a cansaço que sentem, o restante pessoal trata da higiene e vai deitando as crianças.

Mais uma vez, a questão da atenção individualizada é omissa e raramente se coloca a hipótese de divisão do grupo de crianças em grupos mais pequenos - a organização do tempo é feita pensando no grande grupo (e.g., todos escutam uma história, todos vão almoçar, todos brincam sem ou com pouca intervenção do adulto). A única exceção a esta forma de organização prende-se com a realização de pequenos trabalhos para expor ou entregar às famílias; neste caso, a estratégia utilizada pela maioria das educadoras é realizar com cada criança, à vez, esse trabalho individual, numa mesa afastada. Nestes casos, trata-se de uma atividade pouco natural, comandada pelo educador e com muito pouco espaço para a iniciativa e espontaneidade da criança.

Finalmente, no estilo do adulto, é a sensibilidade que se destaca como aspeto mais positivo, relacionando-se com o carinho prestado e a valorização e respeito pelas conquistas individuais das crianças. A maior preocupação é a autonomia dada às crianças, que aparece quase sempre como aspeto a ser estimulado. As nossas observações coincidem com a ideia de que a sensibilidade será o aspeto mais positivo do estilo do adulto, no que diz respeito ao carinho prestado (e.g., gestos de afeto, conforto num momento difícil). Contudo, esta sensibilidade não é, muitas vezes, acompanhada de uma atenção individualizada, pela própria forma como o contexto se organiza, o que torna esta dimensão incompleta e insuficiente. A autonomia é, de 
facto, um aspeto crítico, sendo raros os momentos em que as crianças são incentivadas a desenvolver a sua autonomia, seja em tarefas do quotidiano (e.g., descalçar-se), seja na escolha e no desenvolvimento das atividades. A justificação apresentada para esta lacuna é, geralmente, a falta de tempo e de recursos humanos, já que a criança necessita de muito mais tempo para realizar as suas conquistas do que o tempo que leva a um adulto calçá-la ou alimentá-la, por exemplo.

Algumas questões emergem desta análise dos fatores contextuais. Em primeiro lugar, a oferta educativa aparece como o fator mais destacado e aprofundado por quase todas as educadoras. É notório que esta é uma área central da sua prática, verificando-se um posicionamento em que a qualidade do trabalho pedagógico é lido pela existência ou inexistência de materiais ou equipamentos, em grande parte, elementos extrínsecos ao próprio educador. Isto também se verifica nas outras áreas: as limitações ou obstáculos são geralmente justificados com recurso a fatores externos (falta de materiais, de recursos humanos, de abertura por parte da direção, etc.) e não assumidos como falhas da própria educadora. Podemos estabelecer uma relação entre esta atribuição de causalidade externa e o facto de haver uma tendência para as educadoras se referirem a si na primeira pessoa quando apresentam aspetos positivos, mas optarem pelo termo "nós" (a equipa), quando apresentam obstáculos ou limitações. Este é, contudo, um aspeto que requer uma análise mais aprofundada.

Em segundo lugar, há uma forte correspondência entre a análise das educadoras e as da investigadora, o que leva a crer que os grandes temas são evidentes tanto para um elemento interno como para um externo. Mesmo que de forma implícita, é possível perceber nas respostas das educadoras alguns temas mais problemáticos. A presença de um elemento externo levará, muitas vezes, a alguns comportamentos menos naturais e a respostas em função da desejabilidade social, pelo que a comparação entre as duas visões se revela particularmente útil.

\section{Discussão e considerações finais}

Apesar das críticas apontadas às abordagens centradas nas práticas (e.g., suposta falta de rigor e validade e impossibilidade de generalização), o seu contributo é significativo para o campo da avaliação e da investigação, permitindo o acesso a informações inacessíveis de outro modo. Embora reconheçamos que o material empírico não garante um acesso direto ao significado e que a visão demasiado "de dentro" pode ser, por vezes, pouco válida (Schwandt \& Burgon, 2006) consideramos que esta proximidade é necessária para acompanhar os profissionais de uma forma que permita recolher o retrato o mais fiel possível da sua realidade quotidiana. 
Os resultados apresentados fornecem-nos um primeiro vislumbre do tipo de avaliação que pode ser desenvolvida com recurso a uma ferramenta como o CRECHEndo, permitindo compreender a perspetiva das educadoras de infância participantes sobre o seu contexto e sobre a interação do grupo de crianças com o mesmo. Todos os resultados apresentados se revestem de um caráter preliminar, exigindo análises mais aprofundadas e a triangulação dos diversos dados recolhidos. Embora não seja possível retirar dos resultados agora apresentados a evidência de mudanças nos diferentes contextos, é possível perceber a potencialidade do CRECHEndo enquanto ferramenta de apoio à reflexão do educador sobre a sua prática. A interpretação dos registos efetuados pelas educadoras exigiu um cruzamento com os resultados da observação, de forma a colocar em evidência os possíveis significados subjacentes. Isto significa que muitas das respostas carecerão ainda de uma reflexão mais profunda, sendo também de considerar o facto de algumas educadoras procurarem responder de forma artificial. Uma maior compreensão das potencialidades do CRECHEndo exigirá uma análise mais aprofundada de outros resultados, nomeadamente, a reflexão das educadoras sobre o processo e a comparação dos registos ao longo do tempo. A análise, em profundidade, de cada um dos casos estudados trará também informações mais sólidas sobre a mudança de práticas e os fatores pessoais, interpessoais e institucionais relevantes na implementação de um sistema de avaliação deste tipo.

Parece verificar-se, nesta análise preliminar, uma preocupação das educadoras em preservar uma imagem positiva de si mesmas enquanto profissionais. Isto pode dever-se tanto à falta de sentido crítico sobre o seu trabalho como à necessidade de transmitirem uma imagem favorável da sua prática a um elemento externo. Estes factos vão ao encontro de outros estudos que mostram a tendência das educadoras para atribuírem pontuações mais elevadas ao seu trabalho e mais baixas a fatores externos não controláveis por elas (Sheridan, 2000). Independentemente destas considerações, devemos colocar também a hipótese de que a designação "análise do contexto" possa remeter, erroneamente, para algo externo e separado do educador, pelo que este é um aspeto que deverá ser melhorado.

Utilizada de uma forma contínua, e em articulação com as futuras OPC, esta ferramenta oferece a possibilidade de os educadores em creche investirem na avaliação direcionada para a ação e para a melhoria, numa perspetiva de baixo para cima (ou seja, atendendo às experiências internas das crianças). De uma forma acessível e sistematizada, os educadores podem averiguar os progressos do grupo como um todo e de cada criança individualmente, interpretando-os como reflexo de um conjunto de fatores externos e internos aos mesmos. A partir desta reflexão, torna-se mais fácil estabelecer objetivos e delinear estratégias concretas. 
A concluir, importa acrescentar que o objetivo comum a todos os estudos passa por contribuir para a produção de conhecimento relevante para a comunidade científica e profissional. Neste artigo, procurámos evidenciar os temas emergentes da avaliação dos educadores sobre os seus contextos, contribuindo para a construção de conhecimento sobre o contexto educativo de creche em Portugal. Consideramos que a creche ainda está longe de ser um espaço educativo reconhecido, associando-se mais a preocupações de natureza social e assistencial e estando o trabalho do educador de infância muitas vezes assoberbado pelas rotinas de cuidados (não articuladas com a sua dimensão educativa). Neste sentido, o adulto na creche parece assumir-se mais como um adulto-cuidador, que acarinha e cuida, que um adulto-educador que tem consciência que, ao mesmo tempo que acarinha e cuida, estimula e incita a curiosidade. As preocupações com a autonomia das crianças levam-nos a crer que há ainda um longo caminho a percorrer no sentido de conceber a criança como competente, equilibrando de forma harmoniosa o seu espaço para a iniciativa com a necessidade de proteção do adulto. Estas e outras hipóteses deverão ser aprofundadas em trabalhos futuros, contribuindo para a consolidação de mudanças progressivas nas conceções e práticas da educação de infância em Portugal.

\section{Referências bibliográficas}

Aguiar, C., Bairrão, J., \& Barros, S. (2002). Contributos para o estudo da qualidade em contexto de creche na área metropolitana do Porto. Revista Infância e Educação, 5, 7-28.

Araújo, S. (2012a). Pedagogia em creche: Da avaliação da qualidade à transformação praxiológica (Tese de doutoramento não publicada). Universidade do Minho, Braga.

Araújo, S. (2012b). Researching change: A praxeological case study on toddlers' educational contexts. European Early Childhood Education Research Journal, 20(4), 505-517. doi:10.1080/1350293X.2012.737706

Bagnato, S., \& Neisworth, J. (2007). Assessment for early intervention: Best practices for professionals. New York: The Guilford Press.

Barros, S. (2007). Qualidade em contexto de creche: Ideias e práticas (Tese de doutoramento não publicada). Universidade do Porto, Porto. Consultado em http://hdl.handle. net/10216/19498

Barros, S., \& Aguiar, C. (2010). Assessing the quality of Portuguese child care programs for toddlers. Early Childhood Research Quarterly, 25(4), 527-535. doi: 10.1016/j. ecresq.2009.12.003

Bertram, T., \& Pascal, C. (2009). Desenvolvendo a qualidade em parcerias. Lisboa: Ministério da Educação - DGIDC.

Carvalho, C. (2012). «CRECHEndo» com qualidade: Construção de um instrumento de avaliação das práticas educativas em creche (Dissertação de mestrado não publicada). Universidade de Coimbra, Coimbra. 
Castilho, A., \& Rodrigues, P. (2013). Práticas de avaliação em educação de infância: Evidências de uma investigação naturalista em três jardins de infância diferentes. In M. J. Cardona \& C. Guimarães (Eds.), Avaliação na educação de infância (pp. 78-96). Viseu: Psicossoma.

Coelho, A. (2004). Educação e cuidados em creche: Conceptualizações de um grupo de educadoras (Tese de doutoramento não publicada). Universidade de Aveiro, Aveiro.

Craveiro, C. (2011). Escutando educadoras de infância em creche. In CNE (Ed.), Educação da criança dos 0 aos 3 anos (pp. 93-106). Porto: Conselho Nacional de Educação.

Cryer, D. (1999). Defining and assessing early childhood program quality. The ANNALS of the American Academy of Political and Social Science, 563(1), 39-55. doi:10.1177/000271629956300103

Decreto-Lei n² 241/2001 de 30 de agosto. Diário da República nº. 201/2001 - I Série A. Lisboa: Ministério da Educação.

Estrela, M. T., \& Estrela, A. (2001). (Eds.). IRA: Investigação, reflexão, acção e formação de professores - estudos de caso. Porto: Porto Editora.

Estrela, M. (2008). Qualidade da oferta educativa na creche (Dissertação de mestrado não publicada). Universidade de Aveiro, Aveiro. Consultado em http://hdl.handle. net/10773/1024

Góis, S., \& Portugal, G. (2009). A avaliação da qualidade num contexto de educação de infância. In G. Portugal (Ed.), Ideias, projectos e inovação no mundo das infâncias: O percurso e a presença de Joaquim Bairrão (pp. 213-228). Aveiro: Universidade de Aveiro.

Gullo, D. (2014). Alternative means of assessing children's learning in early childhood classrooms. In B. Spodek \& O. Saracho (Eds.), Handbook of research on the education of young children (pp. 443-455). London: Routledge.

Hoffman, J. (2013). Avaliação mediadora na educação infantil. Em M. J. Cardona \& M. Guimarães (Ed.), Avaliação na educação de infância (pp. 254-266). Viseu: Psicossoma.

Kalliala, M., \& Tahkokallio, L. (2003). The adult role in Finnish early childhood education and care. In F. Laevers \& L. Heylen (Eds.), Involvement of children and teacher style: Insights from an international study on experiential education. Studia Pedagogica 35 (pp. 93-109). Leuven: Leuven University Press.

Laevers, F. (1997). Assessing the quality of childcare provision: «Involvement» as criterion. Researching Early Childhood, 3, 151-165.

Laevers, F. (2000). Forward to basics! Deep-level-learning and the experiential approach. Early Years: An International Research Journal, 20(2), 20-29. Consultado em http:// www.tandfonline.com/doi/abs/10.1080/0957514000200203

Laevers, F. (2005a). Deep level learning and the experiential approach in early childhood and primary education. Leuven: Leuven University Department of Educational Sciences.

Laevers, F. (2005b). Sics [Ziko] Well-being and involvement in care: A process-oriented self-evaluation instrument for care settings. Leuven: Kind \& Gezin and Research - Centre for Experiential Education, Leuven University. Consultado em http://www. kindengezin.be/img/sics-ziko-manual.pdf

Laevers, F. (2014). Fundamentos da educação experiencial: Bem-estar e envolvimento na educação infantil. Estudos em Avaliação Educacional, 25(58), 152-185. doi: http:// dx.doi.org/10.18222/eae255820142794 
Laevers, F., \& Declercq, B. (2011). Raising levels of well-being and involvement in Milton Keynes Preschool Settings [School year 2009-2010]. Leuven: CEGO. Consultado em http://childrenscentre.pengreen.org/wp-content/uploads/sites/2/2014/12/ Milton-Keynes-report.pdf

Laevers, F., Moons, J., \& Declercq, B. (2012). A process-oriented monitoring system for the early years [POMS]. Leuven: CEGO.

Lopes da Silva, I. (2013). Problemas e dilemas da avaliação em educação de infância. In M. J. Cardona \& M. Guimarães (Eds.), Avaliação na educação de infância (pp. 151170). Viseu: Psicossoma.

Machado, I. (2014). Avaliação da qualidade em creche: Um estudo de caso sobre o bem-estar das crianças (Dissertação de mestrado não publicada). Universidade do Minho, Braga. Consultado em http://hdl.handle.net/1822/30211

Moreno, A., \& Klute, M. (2011). Infant-toddler teachers can successfully employ authentic assessment: The learning through relating system. Early Childhood Research Quarterly, 26(4), 484-496. doi:10.1016/j.ecresq.2011.02.005

Nabuco, M., \& Prates, S. (2003). Improving the quality of early childhood education from «within». In F. Laevers \& L. Heylen (Eds.), Involvement of children and teacher style: Insights from an international study on experiential education. Studia Pedagogica 35 (pp. 43-59). Leuven: Leuven University Press.

Nóvoa, A. (1991). Concepções e práticas de formação contínua de professores. In J. Tavares, J. Moreira, \& R. Oliveira (Eds.), Formação contínua de professores: Realidades e perspectivas (pp. 15-38). Aveiro: Universidade de Aveiro.

OCDE. (2014). Proposal for key principles of a quality framework for early childhood education and care. Consultado em http://ec.europa.eu/education/policy/strategic-framework/ archive/documents/ecec-quality-framework_en.pdf

Oliveira, F., \& Gaspar, M. F. (2004). Olhares sobre a avaliação em educação pré-escolar. Revista Portuguesa de Pedagogia, 38(1, 2, 3), 451-484.

Oliveira-Formosinho, J. (2001). A profissionalidade específica da educação de infância e os estilos de interacção adulto-criança. In J. Oliveira-Formosinho (Ed.), Associação Criança: Um contexto de formação em contexto (pp. 80-103). Braga: Livraria Minho.

Oliveira-Formosinho, J. (2002). A avaliação alternativa na educação de infância. In J. Oliveira-Formosinho (Ed.), A supervisão na formação de professores: Da sala à escola (pp. 144-157). Porto: Porto Editora.

Pascal, C., \& Bertram, T. (2003). The effective early learning project: The quality of adult engagement in early childhood settings in the UK. In F. Laevers \& L. Heylen (Eds.), Involvement of children and teacher style: Insights from an international study on experiential education. Studia Pedagogica 35 (pp. 77-91). Leuven: Leuven University Press.

Portaria n. ${ }^{\circ}$ 262/2011 de 31 de agosto. Diário da República no. 167/2011 - I Série. Lisboa: Ministério da Solidariedade e da Segurança Social.

Portugal, G. (2012a). Finalidades e práticas educativas em creche: Das relações, actividades e organização dos espaços ao currículo na creche. Porto: CNIS.

Portugal, G. (2012b). Uma proposta de avaliação alternativa e «autêntica» em educação pré-escolar: O Sistema de Acompanhamento das Crianças (SAC). Revista Brasileira de Educação, 17(51), 593-744. doi:10.1590/S1413-24782012000300006 
Portugal, G. (2013). Avaliar o desenvolvimento e a aprendizagem das crianças: Desafios e possibilidades. In M. J. Cardona \& C. M. Guimarães (Eds.), Avaliação na educação de infância (pp. 234-253). Viseu: Psicossoma.

Portugal, G., Bento, G., \& Carvalho, C. (2016). Orientações pedagógicas para a creche. Documento em apreciação pública.

Portugal, G., \& Laevers, F. (2010). Avaliação em educação pré-escolar: Sistema de Acompanhamento das Crianças. Porto: Porto Editora.

Santos, P., \& Portugal, G. (2002). Avaliação processual da qualidade em educação: Um contributo experiencial para uma escola inclusiva. In J. Costa, A. Neto-Mendes, \& A. Ventura (Eds.), Avaliação de organizações educativas: Actas / II Simpósio sobre Organização e Gestão Escolar. Aveiro: Universidade de Aveiro.

Schwandt, T., \& Burgon, H. (2006). Evaluation and the study of lived experience. In F. Shaw, J. Greene, \& M. Mark (Eds.), Handbook of evaluation: Policies, programs and practices (pp. 98-117). London: Sage.

Sheridan, S. (2000). A comparison of external and self-evaluations of quality in early childhood education. Early Child Development and Care, 164, 63-78. doi: http://dx.doi. org/10.1080/0300443001640106

Shonkoff, J., \& Phillips, D. (2000). From neurons to neighborhoods: The science of early childhood development. Washington, D. C.: National Academy Press.

Taguma, M., Lijtens, I., \& Makowiecki, K. (2012). Quality matters in early childhood education and care - Portugal. Paris: OCDE.

Ulich, M., \& Mayr, T. (2003). Implementing the involvement scales in German day care centers: Practitioners perspectives. In F. Laevers \& L. Heylen (Eds.), Involvement of children and teacher style: Insights from an international study on experiential education. Studia Pedagogica 35 (pp. 25-41). Leuven: Leuven University Press.

UNICEF. (1989). A Convenção sobre os Direitos da Criança. New York: UNICEF. Consultado em https:// www.unicef.pt/docs/pdf_publicacoes/convencao_direitos_crianca2004.pdf

Vandell, D., \& Wolfe, B. (2000). Child-care quality: Does it matter and does it need to be improved? (IRP Special Report n. 78). Madison: University of Wisconsin, Institute for Research on Poverty.

Van Sanden, P., \& Joly, A. (2003). Well-being and involvement as a guide in realizing good conditions for inclusive education in Nicaragua. In F. Laevers \& L. Heylen (Eds.), Involvement of children and teacher style: Insights from an international study on experiential education. Studia Pedagogica 35 (pp. 143-172). Leuven: Leuven University Press.

Vasconcelos, T. (1997). Ao redor da mesa grande: A prática educativa de Ana. Porto: Porto Editora.

Vasconcelos, T. (2009). Prática pedagógica sustentada: Cruzamento de saberes e de competências. Lisboa: Edições Colibri/Instituto Politécnico de Lisboa.

Vasconcelos, T. (2011). Recomendação n. 3/2011 de 21 de abril. Diário da República nº 79/2011 - Il Série. Lisboa: Conselho Nacional de Educação.

Vieira, A., \& Sarmento, T. (2009). Formação em contexto: Uma experiência no jardim de infância São Lázaro/ Braga, Portugal. Actas do X Congresso Internacional Galego-Português de Psicopedagogia (pp. 1057-1067). Braga: Universidade do Minho. 


\section{Normas de Colaboração}

\section{Normas de formatação}

1.1. A Revista Portuguesa de Pedagogia publica artigos científicos em português, inglês e espanhol.

1.2. Os originais deverão ser submetidos em formato word, folha $A 4$ com margens superior, inferior e laterais a 2,54 cm, alinhamento do texto justificado, com avanço de 1,27 cm na primeira linha da cada parágrafo, com espaço duplo entre linhas e com o tipo de letra Times New Roman a 12 pt. Os artigos não devem exceder as 7.000 palavras.

O título do artigo deverá ter alinhamento à esquerda ou justificado, a negrito, letra tamanho 16 pt e todas as palavras principais iniciadas com maiúscula.

Os subtítulos, sem qualquer numeração, deverão ser formatados de acordo com os exemplos seguintes:

\begin{tabular}{|l|l|}
\hline Subtítulo & Formatação \\
\hline nível 1 & Alinhado à esquerda, a negrito, letra tamanho 14 pt, espaço de 6 pt depois \\
\hline nível 2 & Alinhado à esquerda, a negrito, letra tamanho 13 pt, espaço de 6 pt depois \\
\hline nível 3 & Alinhado à esquerda, normal, letra tamanho 12,5 pt, espaço de 6 pt depois \\
\hline nível 4 & Alinhado à esquerda, normal, letra tamanho 12 pt, espaço de 6 pt depois \\
\hline
\end{tabular}

1.3. Acompanhando o original, deverão ser enviados três ficheiros que incluam:

- Folha A: título do artigo; nome, email e enquadramento institucional do(s) autor(es); endereço completo (incluindo telefone, fax e e-mail) do autor responsável por toda a correspondência relacionada com o manuscrito;

- Folha B: título, resumo e palavras-chave em português, inglês e espanhol (resumo com máximo de 150 palavras e até 6 palavras-chave);

- Folha C: declaração de compromisso do(s) autor(es) em como o artigo submetido à Revista Portuguesa de Pedagogia é original, não foi publicado anteriormente e não está submetido para avaliação em qualquer outra publicação científica. 
1.4. A identificação dos autores não deverá constar em nenhum ponto do manuscrito, à exceção da Folha $\mathbf{A}$.

1.5. Os quadros e figuras deverão ser colocados na localização correspondente à sua referência e ordenados em numeração árabe, devendo ser referidos através dessa numeração no texto do artigo, por exemplo: "...cf. figura 10..." e não: "...na figura seguinte...".

1.6. Os quadros e as figuras devem ser formatados de acordo com as Normas da American Psychological Association (APA), 6. a edição, não podendo exceder as dimensões $11,5 \times 18,5 \mathrm{~cm}$ e devem ser entregues no ficheiro do programa em que foram editados originalmente. Essa edição deve ser preparada para impressão a preto e branco. As imagens ou fotografias devem ser entregues em digitalizações de $100 \%$ a 300 dpi's de resolução.

1.7. As referências bibliográficas serão integradas no texto de acordo com o sistema autor-data (consultar normas de publicação da APA). Em caso de citações textuais, acrescentar-se-á o número da página. Exemplos: Piaget afirmou que "a inteligência." (1966, p. 247).

Mas, Zazzo (1972) considera. Diversos autores (Farr \& Moscovici, 1984; Lewin et al., 1944)...

1.8. A lista de referências bibliográficas deverá obedecer às normas de publicação da American Psychological Association (APA), 6a Edição. Exemplos:

\section{a) Livro}

Reuchlin, M. (1992). Introduction à la recherche en psychologie. Paris: Éditions Nathan.

\section{b) Livro traduzido}

Fuller, P. (1983). Arte e psicanálise (M. J. Gomes, Trad.). Lisboa: Publicações Dom Quixote. (Obra original publicada em 1980). No corpo do artigo deve referir-se: Fuller (1980/1983)...

\section{c) Capítulo de livro}

Martin, R. P. (1991). Assessment of social and emotional behavior. In B. A. Bracken (Ed.), The psychoeducational assessment of preschool children (pp. 450-464). Boston: Allyn \& Bacon. 


\section{d) Artigo}

Schein, E. H. (1990). Organizational culture. American Psychologist, 45, 109-119.

\section{e) Comunicação em reunião científica}

Sternberg, R. J. (1997, Setembro). Successful intelligence: What is it, how can it be measured, and can it be taught? Comunicação apresentada na 4th European Conference on Psychological Assessment, Lisboa.

\section{f) Dissertação / Tese}

\section{- Dissertação / Tese não publicada}

Lima, M. P. (1997). NEO-PI-R, Contextos teóricos e psicométricos: "OCEAN" ou "iceberg"? (Tese de doutoramento não publicada). Universidade de Coimbra, Coimbra.

\section{- Dissertação / Tese disponível na Internet}

Bruckman, A (1997). MOOSE Crossing: Construction, community, and learning in a networked virtual world for kids (Tese de doutoramento, Massachusetts Institute of Technology). Consultado em http://www-static.cc.gatech.edu/-asb/thesis/

\section{g) Referências de artigos online}

Hsu, C.-C., \& Sandford, B. A. (2007). The Delphi Technique: Making Sense of Consensus. Pratical Assessment Research \& Evaluation, 12(10). Consultado em Janeiro, 2012, em http://pareonline.net/getvn.asp?v=12\&n=10.

Para os casos não considerados nestes exemplos, os autores devem consultar as normas de publicação da American Psychological Association (APA), 6. edição.

\section{Disposições gerais}

2.1. A Revista Portuguesa de Pedagogia apenas aceita trabalhos originais, que não tenham sido publicados anteriormente e que não estejam submetidos para avaliação em qualquer outra publicação científica.

2.2. Os artigos publicados são da exclusiva responsabilidade dos respetivos autores. A Comissão de Redação reserva-se o direito de não publicar os originais a que a Comissão Científica não atribua nível adequado ou que não obedeçam às normas acima referidas. Os originais não publicados não serão devolvidos. 
2.3. Os originais aceites para publicação ficam propriedade editorial da Revista.

Qualquer reprodução integral ou parcial dos mesmos apenas pode ser efetuada após autorização escrita da Comissão de Redação.

2.4. A Revista Portuguesa de Pedagogia oferece um exemplar de cada número ao 1. autor de cada artigo que o integra.

2.5. Os artigos publicados ficam disponíveis on-line, em acesso livre, através do endereço http://impactum.uc.pt/ 